\title{
Jejunal carcinoma associated with non-absorbable suture material
}

\author{
D.J. Leinhardt, P.J. Smart, ${ }^{1}$ and J.M.T. Howat
}

Departments of Surgery and ${ }^{1}$ Pathology, North Manchester General Hospital, Delauneys Road, Manchester $M 86 R B, U K$.

\begin{abstract}
Summary: A case of adenocarcinoma of the jejunum arising at the site of non-absorbable surgical material is reported. This appears to be a unique observation.
\end{abstract}

\section{Introduction}

Adenocarcinoma of the small bowel accounts for less than $1 \%$ of gastrointestinal malignancies. Many arise de novo; others may be associated with villous adenomata, Peutz-Jegher's syndrome, Crohn's disease, coeliac disease and non-tropical sprue. ${ }^{1}$

We report an apparently unique patient in whom an adenocarcinoma of the jejunum appears to have arisen in association with non-absorbable surgical suture material.

\section{Case report}

A 54 year old man presented with a 2-month history of marked weight loss and profuse, painless vomiting of undigested food. Twenty years earlier he had been treated for a duodenal ulcer by vagotomy and gastroenterostomy following which he had remained symptom-free until his current illness. On examination he was thin and dehydrated. There was a marked gastric succussion splash. A barium meal showed a dilated stomach with retention and stasis of food but the pylorus appeared normal. The duodeno-jejunal flexure was obstructed by a shouldered lesion. Extensive food residue made gastroscopy unsatisfactory. At laparotomy the obstruction was found immediately distal to a retrocolic gastrojejunostomy where lay an indurated ulcer. The stoma was dismantled, the greater curve of the stomach repaired, and the involved segment of jejunum resected. On opening the specimen a $2 \mathrm{~cm}$ length of non-absorbable suture material (Courlene X3) with small calculi adherent to it was found projecting from the centre of the ulcer. Histology showed a well differentiated adenocarcinoma (Figure 1). Postoperatively the patient remained well for 12 months when he

Correspondence: J.M.T. Howat, M.D., F.R.C.S.

Accepted: 24 March 1988

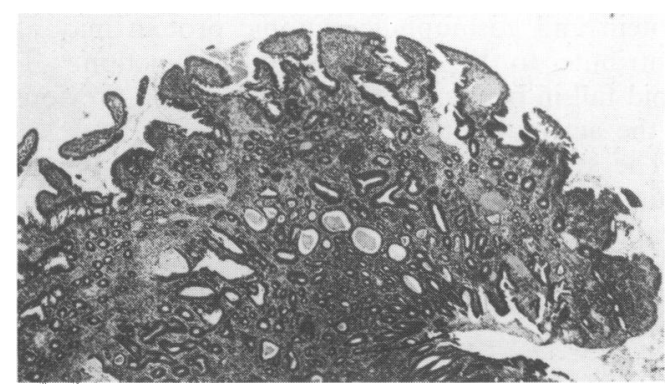

Figure 1 Histological appearances of the jejunal lesion $(25 \times$ magnification $)$.

became anaemic and was found to have two further, distinctly separate, adenocarcinomas of the right colon. At extended right hemicolectomy there was no sign of local recurrence of the original jejunal tumour.

\section{Discussion}

Following gastric surgery there is a small increase in the incidence of carcinoma of the gastric remnant. In this case, however, the tumour was distal to the anastomosis. Recurrent benign ulcers, which occur in $1-5 \%$ of patients who have had previous peptic ulcer surgery, are commonly sited on the distal aspect of the anastomosis and although there is some risk of malignancy arising in a gastric ulcer, ${ }^{2}$ this has not been reported in an anastomotic ulcer.

Non-absorbable sutures may be recognized at an anastomosis during endoscopy in patients who have developed recurrent symptoms after gastric surgery. In many of these cases there is no associated ulceration, but should ulceration develop, suture material often protrudes from the centre of it. ${ }^{3}$

Malignant tumours, usually sarcomas or lymphomas, can be induced by surgical implants; silicone or metal in orthopaedic procedures, ${ }^{4,5}$

(C) The Fellowship of Postgraduate Medicine, 1988 
silicone injections in cosmetic breast surgery ${ }^{6}$ and by plastic arterial prostheses. ${ }^{7}$ Colonic adenocarcinoma has been found in association with intraluminal foreign bodies. ${ }^{8}$

Suture line recurrence is well recognized in the surgery of colonic tumours and although this is usually thought to be due to inadequate surgery rather than tumour arising de novo, stainless steel sutures have been implicated in the localization and development of chemically induced tumours in the rat colon. ${ }^{9}$

Courlene X3, a monofilament of high density polyethylene, was commonly used in the manufacture of ropes and fishing nets. In an undyed form it enjoyed a vogue as a suture material with a small number of surgeons in the 1960s. The advantages claimed for it included cheapness, high tensile strength and ease of use as a continuous suture. Short term studies showed it to be almost inert, causing minimal cellular reaction in both animal and human tissues. ${ }^{10}$ It contained neither plasticisers or stabilisers (Courtaulds, personal communication).

Although it cannot be proved that the suture induced the jejunal carcinoma in our patient, it seems highly probable that it did. While the persistence of the suture ( $>20$ years) may have

\section{References}

1. Williamson, R.C.N., Welch, C.E. \& Malt, R.A. Adenocarcinoma and lymphoma of the small intestine: distribution and etiologic associations. Ann Surg 1983, 197: 172-178.

2. Farini, F., Farinati, F., Cardin, F. et al. Evidence of gastric carcinoma during follow-up of apparently benign gastric ulcer. Gut 1983, 24: A486.

3. Hoare, A.M. \& Alexander-Williams, J. Thread sutures seen on gastroscopy, do they cause ulcers or indigestion? $\mathrm{Br}$ Med J 1977, ii: 996-997.

4. Digby, J.M. \& Wells, A.L. Malignant lymphoma with intranodal refractile particles after insertion of silicone prosthesis. Lancet 1981, ii: 580.

5. McDonald, I. Malignant lymphoma associated with internal fixation of a fractured tibia. Cancer 1981, 48: 1009-1011.

6. Lewis, C.M. Inflammatory carcinoma of the breast following silicon injections. Plast Reconst Surg 1980, 66: $134-136$. caused a chronic ulcer to develop which subsequently underwent malignant transformation, this sequence of events can perhaps be discounted, as histologically there was no evidence of the acute or chronic inflammatory infiltrate that might have been expected in long-standing ulceration. The alternative possibility exists that the suture may have stimulated the local development of malignant change directly. Such an association has not been previously reported outside of an experimental system. ${ }^{9}$ The subsequent development of two primary colonic tumours in this patient is not without interest. Small bowel adenocarcinomas have been previously recorded in association with malignancies at other sites including the gastrointestinal tract, ${ }^{1}$ and this, together with the recent observation that colonic tumours may be found more frequently in patients who have undergone surgery for peptic ulcer, ${ }^{11}$ serves to emphasize that conditions other than local factors may be important in the development of malignancy.

\section{Acknowledgement}

We are grateful to Mr M.D. Smith of Courtaulds Patent Services for details of Courlene X3.

7. Weinberg, D.S. \& Maini, B.S. Primary sarcoma of the aorta associated with a vascular prosthesis. Cancer 1980, 46: 398-402.

8. Melville, D.M. \& Thompson, M.R. Carcinoma of the sigmoid colon secondary to a foreign body. Br J Surg 1987, 74: 389.

9. Phillips, R.K.S. \& Cook, H.T. Effect of steel wire sutures on the incidence of chemically induced rodent colonic tumours. Br J Surg 1986, 73: 671-674.

10. Haxton, H. Surgical Techniques. John Wright and Sons, Bristol, 1970, pp 67-72.

11. Bundred, N.J., Whitfield, B.C.S., Stanton, E., Prescott, R.A., Davies, G.C. \& Kingsworth, A.N. Peptic ulcer surgery - an important factor in the aetiology of colorectal cancer. Scot Med J 1984, 29: 257. 